\title{
Classification of Glandular Cells using a Pre-trained Convolutional Neural Network
}

\author{
Migyung Cho
}

\begin{abstract}
Tissues can be divided into glandular structures (cells) and non-glandular when we magnify them with a microscope. To classify the two types, we performed experiments using convolutional neural network. We cropped regions of glandular cells and non-glandular in $10 x$ magnification images. The size of cropped images was between 80 and 100 pixels in width and height. We prepared 932 glandular cells and 1000 non-glandular for the train and test. Of these, 1468 were used for learning and 532 were used for testing. We trained and tested the dataset using a slightly modified pre-trained VGG16. The inside of glandular cells consists of nucleus, lumen and cytoplasm. Normal glandular cells and abnormal glandular cells that we call tubular adenoma have different texture features. But both types of glandular cells have distinct boundaries and specific shapes. In the case of cancer, as the nucleus grows excessively, the boundaries of the glandular cells become unclear and disappear. We trained three types of glandular cells, which is normal, tubular adenoma, and cancer. Experimental results using the pre-trained VGG16 classification showed a high classification accuracy of 99.44\%. Only three non-glandular out of the 532 test data were misclassified into glandular cells. The classification method presented in the paper can be used to eliminate false positives that produced by an automatic segmentation system for the pathology image. The performance of the segmentation can be improved by eliminating segmented objects that are false positives.
\end{abstract}

Keywords : Classification, convolution neural network, glandular cells, pathology image, segmentation

\section{INTRODUCTION}

Many researchers have been studying automatic classification systems to support pathologists for cancer grading work [1]-[4]. The purpose of an automatic classification system is to improve the accuracy and consistency of classification by developing computer algorithms that can objectively measure tumor grades. In recent decades, studies have been conducted on the classification of cancer in various tissues such as lung cancer and prostate cancer [3], [4]. The most approach for grading tumor is to analyze morphological features by segmentation of glandular cells [5], [6]. This is why pathologists look at the morphological features of glandular cells to determine whether it is cancer or not.

The segmentation of glandular cells is the basic task of an automated cancer grading system. Many researchers have studied the segmentation for biomedical images [5]-[10]. The

Revised Manuscript Received on July 22, 2019.

* Correspondence Author

Migyung Cho, College of Software Convergence, Tongmyong University, 428, Sinseon-ro, Nam-gu, Busan, 48520, Korea , mgcho@tu.ac.kr

segmentation is a very challenging problem and no automation algorithm with satisfactory accuracy has yet been found. The segmentation methods using the convolutional neural network $(\mathrm{CNN})$ gave relatively better results, but gave the low accuracy when the image was cancer. This is because segmentation results include false positive and false negative objects.

In this paper, we carried out experiments to classify glandular cells and non-glandular using a convolution neural network to remove false positives that were produced by the automatic segmentation. Experiments were performed to classify input cell images into two types of images, which are glandular structures and non-glandular structures, by patterns and texture characteristics. We used a set of cell images extracted from colon tissues. There are only two class types that will be categorized, and there are two thousands of data sets prepared. Two thousands of data sets are not enough to train a convolutional learning model. Therefore, we used the slightly modified pre-trained VGG16 model, which provides simple but relatively high classification accuracy among CNN-based deep neural networks [11].

Our experiments had shown that the pre-trained model should be applied for more accurate classification in the case of the learning data set is small. We cropped areas corresponding to glandular cells and non-glandular in 10 times magnified pathology images, respectively. We prepared 932 glandular cells and 1000 non-glandular data. Experimental results using the pre-trained VGG16 classification model showed a high classification accuracy of $99.44 \%$. Classification model that gave the high accuracy of more than $99 \%$ can remove most of segmented objects, which are false positives, and so it can improve the accuracy of segmentation.

The paper organizes as follows. In Section 2, we look at the characteristics of glandular cells and non-glandular and explain how to prepare dataset from pathological images. Also, in Section 3, we describe the overall architecture of our network model and experimental methods. In Section 4, we analyze the experimental results. We conclude in Section 5

\section{CHARACTERISTICS OF GLANDULAR CELLS AND NON-GLANDULAR}

Digital pathology images scanned from glass slides can generally be magnified up to 50 times. Our study is to classify the images extracted from the colon tissue into glandular cells or non-glandular to improve the performance of the automatic segmentation system. 
We obtained glandular cells and non-glandular data from $10 \times$ magnified pathology images with the help of a pathologist. Normal glandular cells and abnormal glandular cells have different texture features. Let's look at the representative features of glandular cells and non-glandular used in experiments.

Fig. 1 is the partial area of $10 \times$ magnified image of the whole slide image that scanned a glass slide. The yellow area is the glandular cells and area except for glandular cells is the non-glandular, which is called as the stroma area. Fig. 1 shows three glandular cells. We cropped the yellow area to prepare glandular cells data. The non-glandular data was prepared by clipping some parts of the area excluding the yellow area.

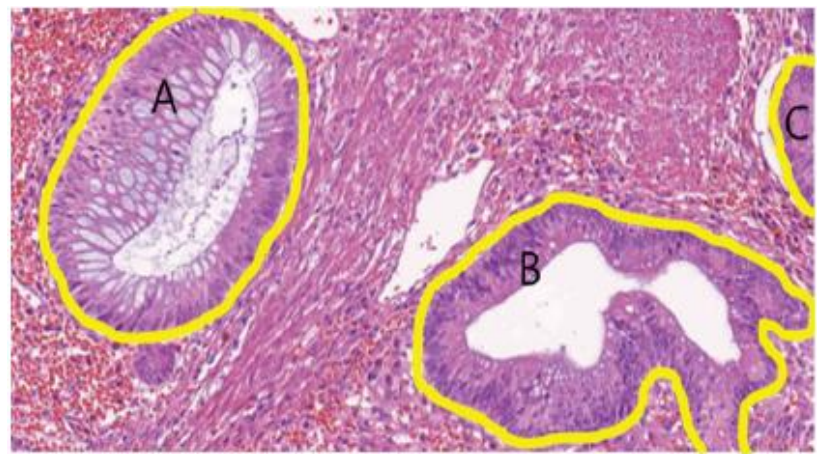

Fig. 1. A, B and C: Glandular cells. Areas except for A, B and C: Non-glandular

\section{A. Glandular cells}

We obtained three kinds of glandular cells that are normal, tubular adenoma, and cancer from a 10 times magnified pathology image with the help of a pathologist. Texture features of normal, tubular adenoma, and cancer glandular cells are very difference from each other. Fig. 2(a) shows examples of normal glandular cells, Fig. 2(b) means examples of tubular adenoma, and Fig. 2(c) is examples of cancer glandular cells. In the case of normal, the cytoplasm is developed and nuclei are located around the cell boundary.

In the case of tubular adenoma, the lumen located in the middle region of the glandular cells becomes wider and the proportion of the nuclei area becomes increased. In the case of cancer, the border of glandular cells is unclear and the nucleus is proliferating disorderly. The size of glandular cells becomes irregular. We had cropped three types of glandular cells from digital pathology images. The cropped images were between 80 pixels and 100 pixels in both width and height. We prepared 932 glandular cells.
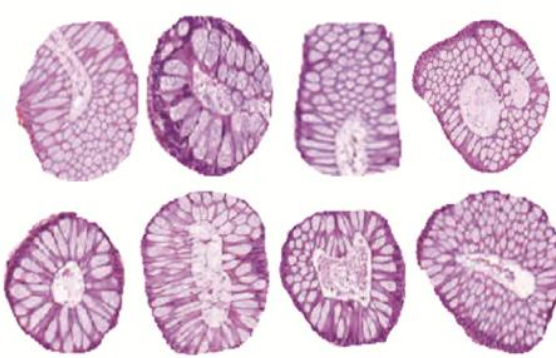
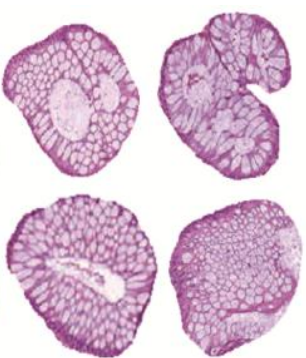

(a)
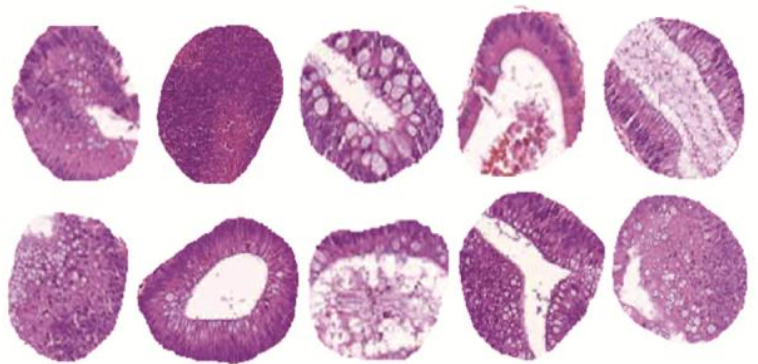

(b)
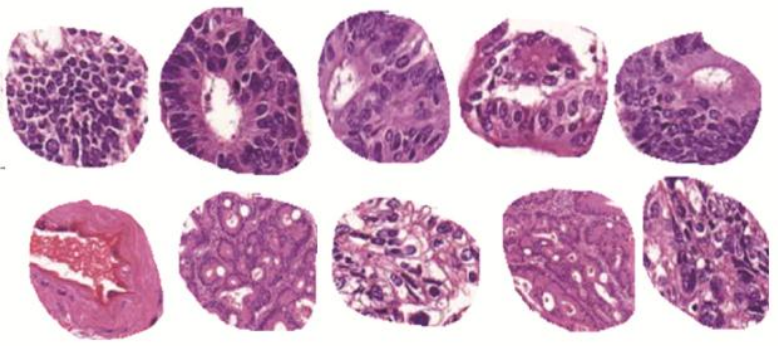

(c)

Fig. 2. Three kinds of glandular cells: (a) Normal (b) Tubular adenoma (c) Cancer

\section{B. Non-glandular}

All regions except for the glandular cells on the pathology image are non-glandular regions. Because they have a wide variety of texture features, they seem to have no rules. Sometimes they have a small number of scattered nuclei. We prepared 1000 non-glandular data sets in the same way as preparing the glandular cells data set. The biggest difference from the glandular cells is that there is no nucleus in the certain direction or that there is no dense proliferating nucleus that appears in cancer. In addition, there is no cytoplasm in normal glandular cells, and there is little clear lumen area that appears frequently in tubular adenoma. But the rightmost data in Fig. 3 shows the area that looks like a lumen.
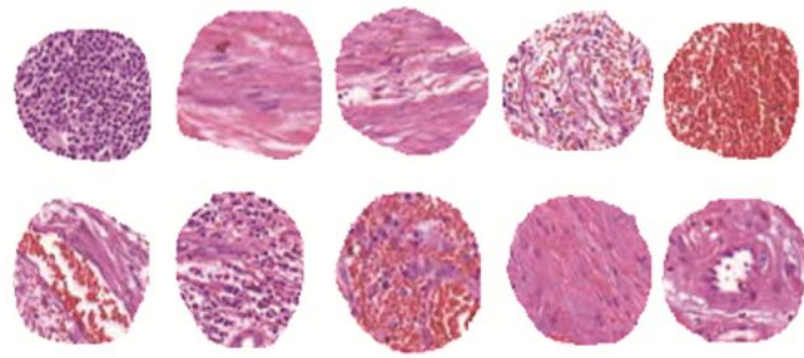

Fig. 3. Various non-glandular areas

\section{NETWORK MODEL FOR CLASSIFICATION}

Representative models for classification are VGG, GoogleNet, and ResNet etc. In particular, GoogleNet and ResNet implemented very deep networks to accurately distinguish various objects. In recent years, these deep neural network models provide classification accuracy that exceeds that of humans. ImageNet used to train those deep neural network models provides more than 1000 data per class for 1000 classes.

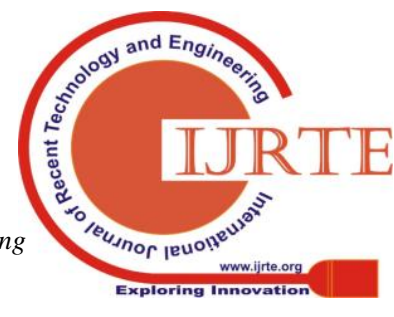


The VGG provided classification accuracy similar to that of the two models, which are GoogleNet and ResNet, although the depth of the network is relatively small [11].

The data we used in our experiments is a small set of hundreds of medical images. We used the VGG16 model because it is simple and provides relatively high classification accuracy. We trained the glandular cells and non-glandular data using the slightly modified pre-trained VGG16 model. We used a pre-trained model to overcome the problems of small data set. Therefore, the filter weights of the VGG16 convolution layer learning of the ImageNet were taken as initial values.

Table I shows slightly modified pre-trained VGG16 we used for learning and testing. The VGG16 model uses the nested $3 \times 3$ convolution in the first and second blocks and 3 times the $3 \times 3$ convolution in the third, fourth, and fifth blocks. At the end of each block, it applies a max pooling. And VGG16 has fully connected layers after convolution layers. In the VGG16 model, the final fully connected layer has a 1 x 1000 vector. Because our problem is classified into two classes, our VGG model has a $1 \times 2$ vector.

The filter weights of the VGG16 convolution layer learning the ImageNet were taken as initial values. We rescaled the size of data because the size of the cropped image is 80 to 100 in width and height, and the input size of VGG16 is $128 \times$ 128. In addition, in order to increase the number of training data, data augmentation techniques such as vertical and horizontal flip and rotation were used. From the total data, 1400 were used as training data and the remaining 532 were used as test data

Table-I: Our model for classification

\begin{tabular}{|c|c|c|c|}
\hline Layer & Type & $\begin{array}{l}\text { Kerne } \\
1 \text { size }\end{array}$ & Maps and neurons \\
\hline 1 & Input & & $\begin{array}{l}1 \text { map of } 128 \times 128 \\
\text { neurons }\end{array}$ \\
\hline 2 & $2 \times$ Convolution & $3 \times 3$ & $\begin{array}{l}64 \text { map of } 128 \times 128 \\
\text { neurons }\end{array}$ \\
\hline 3 & $\begin{array}{l}2 \times \text { Convolution }+ \\
\text { Max pooling }\end{array}$ & $\begin{array}{l}3 \times 3 / \\
2 \times 2 \\
\end{array}$ & $\begin{array}{l}64 \text { map of } 128 \times 128 \\
\text { neurons }\end{array}$ \\
\hline 4 & $\begin{array}{l}3 \times \text { Convolution }+ \\
\text { Max pooling }\end{array}$ & $\begin{array}{l}3 \times 3 / \\
2 \times 2 \\
\end{array}$ & $\begin{array}{l}512 \text { map of } 64 \times 64 \\
\text { neurons }\end{array}$ \\
\hline 5 & $\begin{array}{l}3 \times \text { Convolution }+ \\
\text { Max pooling }\end{array}$ & $\begin{array}{l}3 \times 3 / \\
2 \times 2\end{array}$ & $\begin{array}{l}1024 \text { map of } 32 \times 32 \\
\text { neurons }\end{array}$ \\
\hline 6 & $\begin{array}{l}3 \times \text { Convolution }+ \\
\text { Max pooling }\end{array}$ & $\begin{array}{l}3 \times 3 / \\
2 \times 2\end{array}$ & $\begin{array}{l}512 \text { map of } 16 \times 16 \\
\text { neurons }\end{array}$ \\
\hline 7 & $\begin{array}{l}\text { Fully-connected-2 } \\
048\end{array}$ & & 2048 \\
\hline 8 & $\begin{array}{l}\text { Fully-connected-1 } \\
00\end{array}$ & & 100 \\
\hline 9 & Softmax & & 2 \\
\hline
\end{tabular}

\section{RESULTS AND DISCUSSION}

Fig. 4 shows the learning convergence process of the pre-trained VGG16 we used. Convergence was achieved through 20 epoch very stably. The accuracy of our model were $99.44 \%$ or more. Only three non-glandular areas among total 532 were misclassified into glandular cells. Table II shows the confusion matrix. All glandular cells were classified without error. It means that glandular cells are composed of nucleus, cytoplasm and lumen and have

distinguishable patterns. Non-glandular seems to be more difficult to classify because it has too many texture features.

Table-II: Confusion matrix

\begin{tabular}{|l|l|l|l|}
\hline & $\begin{array}{l}\text { Glandular } \\
\text { cells }\end{array}$ & Non-glandular & $\begin{array}{l}\text { Total } \\
\text { Number }\end{array}$ \\
\hline $\begin{array}{l}\text { Glandular } \\
\text { cells }\end{array}$ & 232 & 0 & 232 \\
\hline $\begin{array}{l}\text { Non-glandular } \\
\text { area }\end{array}$ & 3 & 297 & 300 \\
\hline
\end{tabular}

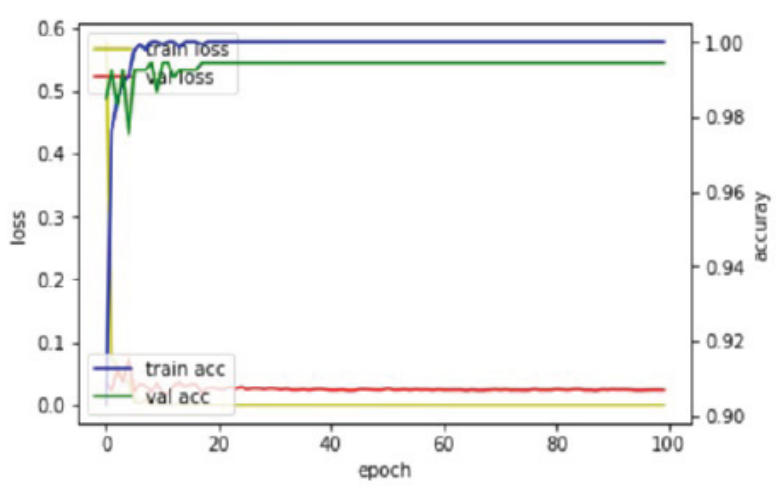

Fig. 4. Loss and accuracy graph

Fig. 5 shows three misclassified non-glandular structures and their probabilities. The fist value means the probability of glandular cells and the other means is the probability of non-glandular. I cannot find a clue as to why three images were misclassified as glands. One of the disadvantages of deep neural network model is that it is not possible to explain why these results came out.
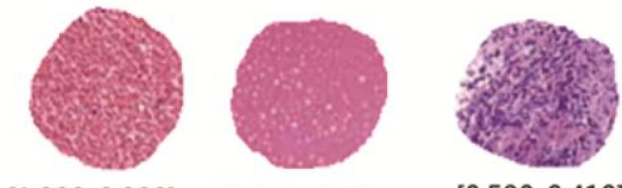

[1.000 0.000]

[0.830 0.170]

[0.590 0.410]

Fig. 5. Three misclassified non-glandular with probability [glands, non-glands]

The classification results presented in the paper can be used to eliminate false positives that were incorrect segmented objects by the automatic segmentation systems. Automatic segmentation system is to find the boundaries of glandular cells. False positives are incorrect segmented objects into glandular cells even though they are non-glandular. Therefore, removing non-glandular from segmented objects can remove false positives from the result of the automatic segmentation. Fig. 6 shows results of segmentation for four pathology images. In the figure, all segmented glandular cells mark in green and the gland cells inside the black rectangles corresponds to false positives. Fig. 6(a) and Fig. 6(b) have one false positive, Fig. 6(c) has two false positives, and Fig. 6(d) has three false positives. Sometimes there is a lot of false positives, which reduces the accuracy of segmentation. 


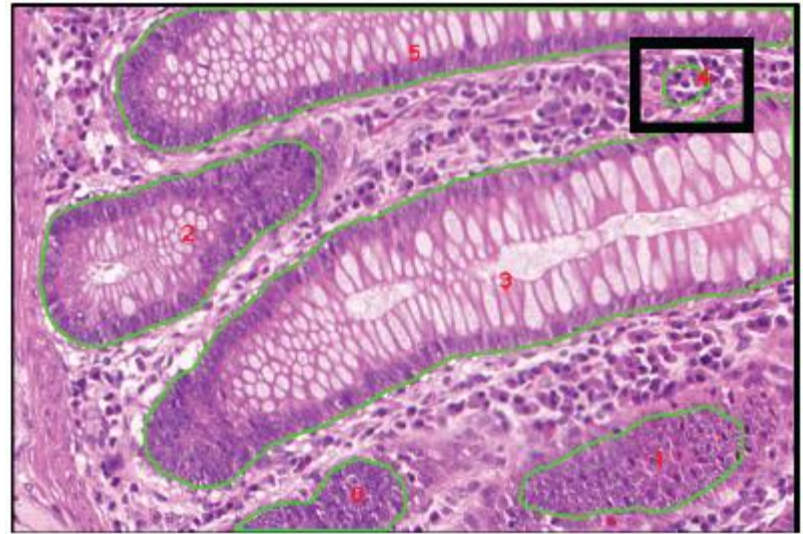

(a)

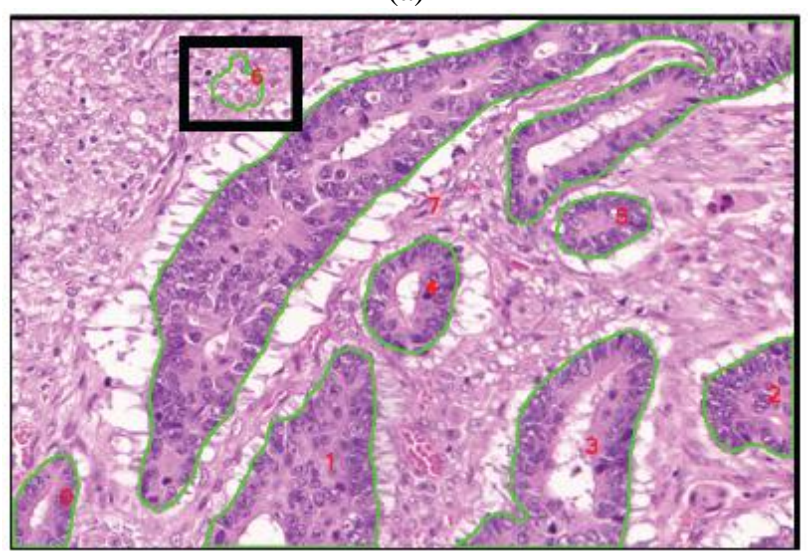

(b)

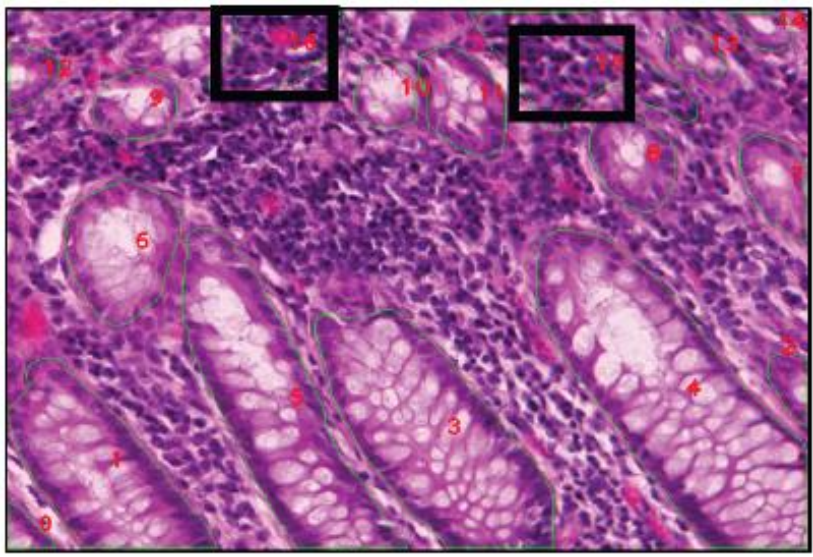

(c)

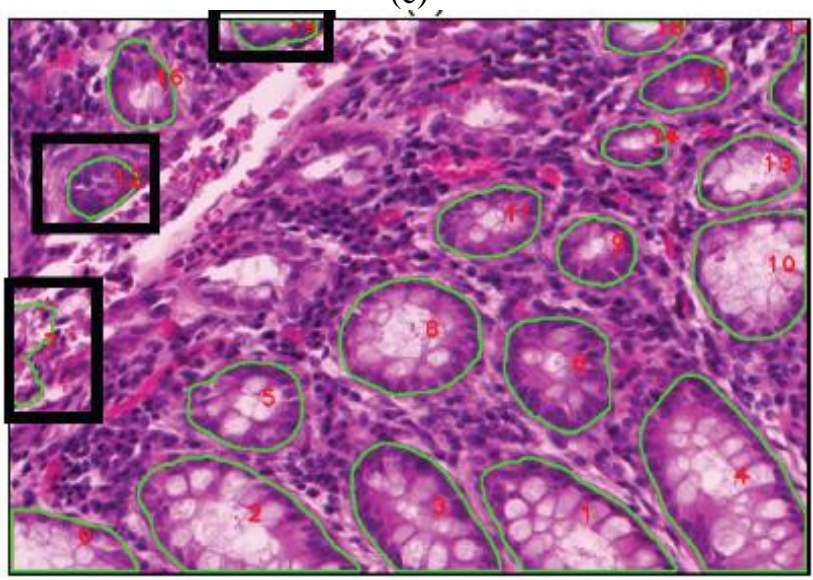

(d)

Fig. 6. Four segmentation results with false positives

To measure the segmentation accuracy, we usually F1score and Diceobj measure. F1score measures the detection accuracy of each gland object. It can be calculated by precision and recall values. Diceobj measures how accurately the segmented objects and ground true objects overlap and it is calculated by the Dice Index. The formula for calculating the precision value takes the denominator of the sum of the true positives and false positives. Therefore, the larger the value of false positive, the lower the value of F1score.

Segmentation accuracy can be improved by eliminating some incorrect segmented glands, which are false positives. We can perform a post-processing step with a $\mathrm{CNN}$ based classification model after segmenting the medical image. We had conducted various experiments that applied the proposed classification model to segmented glandular objects and we found our classification model removed false positives among segmented glandular objects. By removing over $99 \%$ of the false positives, we could increase the total segmentation accuracy, especially, F1score by $1 \%$ on average.

\section{CONCLUSION}

In this paper, we carried out experiments to classify glandular cells and non-glandular using a convolution neural network. We prepared 932 glandular cells and 1000 non-glandular from 10× magnification images. Of these, 1468 were used for learning and 532 were used for testing. For classification, we trained and tested the data using the slightly modified pre-trained VGG16. Experimental results using the pre-trained VGG16 classification showed a high classification accuracy of $99.44 \%$. Only three non-glandular of the 532 test data were misclassified into glandular structures.

The classification method presented in the paper can be used to eliminate false positives, which are incorrect segmented objects produced by the automatic segmentation system of the pathology image. Pathology image consists of areas of glandular cells and non-glands. False positives are incorrect segmented objects into glandular cells even though they are non-glandular. One of the causes of performance degradation of the segmentation algorithm is false positives. We can improve the performance of the automatic segmentation system by eliminating segmented objects that are false positives.

\section{ACKNOWLEDGMENT}

This Research was supported by the Tongmyong University Research Grants 2017(2017A013).

\section{REFERENCES}

1. Adnan Q., Syed M. Anwar, Muhammad M., Muhammad A., Majdi A., "Medical Image Analysis using Convolutional Neural Networks: A Review. Journal of medical systems,” 2018, 42(11), pp. 226.

2. Geert Litjens, Thijs Kooi, Babak Ehteshami Bejnordi, Arnaud Arindra Adiyoso Setio, Francesco Ciompi. Mohsen Ghafoorian, Jeroen A.W.M. van der Laak, Bram van Ginneken, Clara I. S'anchez, "A survey on deep learning in medical image analysis. Medical Image Analysis," 2017, 42, pp.0-88.

3. Wollmann T, Rohr K, “Automatic breast cancer grading in lymph nodes using a deep neural network," 2017, ArXiv170707565 Cs.

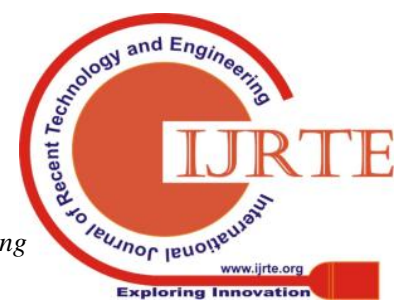


4. Ruqayya Awan et al. "Glandular Morphometrics for Objective Grading of Colorectal Adenocarcinoma Histology Images," Scientific reports. 2017, 7(1), pp. 16852

5. K. Sirinukunwattana, J. P. Pluim, H. Chen, X. Qi, P.-A. Heng, Y. B. Guo, L. Y. Wang, B. J. Matuszewski, E. Bruni, U. Sanchez, et al., "Gland segmentation in colon histology images: The GlaS Challenge Contest," Medical Image Analysis. 2017, 35, pp. 489-502.

6. H. Chen, X. Qi, L. Yu, P. Heng. "Dcan: Deep contouraware networks for accurate gland segmentation," Proc. of the IEEE conference on Computer Vision and Pattern Recognition 2016. 2016, pp. 2487-2496.

7. Migyung cho, Hyekyung Lee, Hwan Gue Cho, "An Automatic Segmentation Algorithm for Colonic Glandular Lesions," Journal of KIISE, 2018, 45(6), pp.554-563.

8. Kayalibay B, Jensen G and van der Smagt P. "CNN-based segmentation of medical imaging data," 2017, arXiv:1701.03056.

9. Kainz P, Pfeiffer M, Urschler M. "Segmentation and classification of colon glands with deep convolutional neural networks and total variation regularization," 2017, https://doi.org/10.7717/peerj.3874.

10. C. Gunduz-Demir, M. Kandemir, A. Tosun, C. Sokmensuer. "Automatic segmentation of colon glands using object-graphs," Medical Image Analysis. 2010, 14(1), pp. 1-12.

11. K. Simonyan and A. Zisserman, "Very deep convolutional networks for large-scale image recognition," International Conference on Machine Learning 2015.

\section{AUTHORS PROFILE}

Migyung Cho received the Ph.D. degree from Pusan National University in 1999. She is currently a professor at College of Software Convergence, Tongmyong University, Busan, Korea. Her research interests is in medical image analysis with interests in pathology images, and algorithm analysis and design for convergence

of software. 